\title{
Assimilative hue shifts in color gratings depend on bar width
}

\author{
CLEMENS FACH and LINDSAY T. SHARPE \\ Albert-Ludwigs-Universität, Freiburg, West Germany
}

\begin{abstract}
Hue shifts were measured in isoluminant color gratings whose bar width was varied from $2^{\prime}$ to $20^{\prime}$ of visual angle. Subjects matched the hues in each grating with individual Munsell swatches. Hue shifts were largest for bar widths of 2'; however, they depended on the color combination used. Green and red shifted toward (i.e., assimilated with) whatever second grating color they were paired with. Blue, on the other hand, assimilated with red and with yellow, but remained relatively unchanged when combined with green. Yellow shifted only minimally, regardless of the second grating color. Hue shifts decreased with increasing stripe width and disappeared between 4.5' and 7.5'. Compared with the assimilative hue shifts, color contrast effects were slight or absent. These results cannot be attributed merely to chromatic aberration, macular pigment, eye movements, or field size.
\end{abstract}

The influence of two or more adjoining chromatic fields on each other's perceived color and lightness has been known for a long time. In fact, as early as 1835 , Chevreul $(1969 / 1835)$ described the subtle color changes introduced by weavers into tapestries to compensate for such effects. After performing extensive experiments to measure these effects on apparent hue and saturation, he was the first to quantify simultaneous color contrast (e.g., red placed next to blue appears yellow). Later, in 1874, von Bezold reported an opposite phenomenon, the so-called spreading effect. He observed this phenomenon in handpainted ornaments in which areas of different colors were interspersed with white and black patterns. Even though the luminance of the colored areas remained constant, they appeared lighter when interspersed with white patterns and darker when interspersed with black patterns. Such changes in perceived lightness are called lightness assimilation, indicating that the lightness of the achromatic pattern has been induced into, or assimilated by, the adjacent portion of color. A more recent term for assimilation appearing in the psychophysical literature is similitude (DeValois \& DeValois, 1975).

In 1962, Helson and Joy reported quantitative measurements of assimilation effects in achromatic gratings. According to their results, assimilation and contrast depend on stripe width, that is, spatial frequency. In high-spatialfrequency gratings, the lightness of white or black bars

This research was supported by the Deutsche Forschungsgemeinschaft, SFB 70, Teilprojekt A6. We thank L. A. Spillmann for his constant advice. We also thank the anonymous referee who gave us the idea for Figure 4. K. Knoblauch, J. Werner, A. Valberg, and V. Volbrecht read and improved an earlier draft of this manuscript. Parts of the manuscript were presented at the spring 1983 meeting of the German Physiological Society, Mainz, FRG, and at the Sixth European Conference on Visual Perception, Lucca, Italy, September 1983.

The authors' address is Albert-Ludwigs-Universităt, Neurologische Klinik, Hansastrasse 9, 7800 Freiburg, West Germany. drawn onto a gray background is assimilated, so that the gray looks lighter when combined with the white grating bars and darker when combined with the black grating bars. With increasing stripe width, this assimilation becomes weaker and ultimately changes into contrast. The gray then looks darker when combined with the white bars and lighter when combined with the black bars. These observations were confirmed by Walker (1978), who reported a critical stripe width of about $8^{\prime}$ of visual angle for the transition from assimilation to contrast.

Assimilative shifts can also be found in color gratings. This was first described by Schober and Munker (1967), who used rectangular color gratings made from papers of different hues, lightnesses, and saturations. The subjects' task was to view the gratings at various distances and to report qualitatively the perceived change in the hue of the grating bars. For large observation distances (i.e., for high spatial frequencies) the subjects reported a shift of the perceived grating colors toward each other (i.e., assimilation). In a red-green grating, for example, the red appeared yellowish-red and the green appeared yellowishgreen. The assimilation effects disappeared at critical stripe widths between $10^{\prime}$ and $30^{\prime}$, depending upon the grating color combination. Wider bars, which might have yielded a transition from assimilation to contrast similar to that found for achromatic gratings, were not studied.

More recently, color contrast was found in an experiment reported by Ware and Cowen (1982). Using a technique adopted from color induction experiments, they measured the hue shifts produced by inducing stripes of $18^{\prime}$ on test stripes of $6^{\prime}$. The colors of the inducing stripes were highly saturated, whereas those of the test stripes were of various lower saturations. Ware and Cowen mainly found color contrast effects. Only in a few conditions did they find assimilation.

It thus seems that the previous studies on assimilation and contrast may be summarized as follows: Both assimi- 
lation and contrast are observed for achromatic gratings, depending upon the stripe width (Helson \& Joy, 1962; Walker, 1978). Coarse gratings show contrast, whereas fine gratings show assimilation. Only assimilative shifts have been reported for color gratings differing in saturation and lightness, provided the duty cycle is equal (Schober \& Munker, 1967). However, if the duty cycle is unequal, then color contrast, induced by the wider stripes onto the smaller ones, can be obtained (Ware \& Cowen, 1982).

These findings prompted the following four questions. First, do both assimilative and contrast color interactions occur between grating stripes of equal duty cycle that have equal lightness and saturation? Second, if so, what are the magnitudes of the perceived hue shifts? Third, do such hue shifts depend on stripe width? Fourth, is the amount of the shift specific to the grating color combination used?

\section{METHOD}

\section{Apparatus and Stimuli}

Square-wave gratings of equal duty cycle were made up of four different Munsell papers: 5R (red), 5Y (yellow), 5G (green), and 5B (blue). The papers had dominant wavelengths for CIE standard illuminant $C$ of $648 \mathrm{~nm}, 579 \mathrm{~nm}, 514 \mathrm{~nm}$, and $441 \mathrm{~nm}$, respectively, as calculated from the $\mathrm{x}-\mathrm{y}$ coordinates given in Wyszecki and Stiles (1982). They were all approximately equal in lightness (value 6) and saturation (chroma 6). Chroma 6 was the highest chroma notation that could be used while maintaining the grating colors at the same lightness level. All six grating combinations were constructed.

The gratings were illuminated by a MACBETH daylight lamp (Type BBX-324, Executive) whose central axis of incidence was at an angle of $45^{\circ}$ from the normal to the grating surface. The lamp was operated at a color temperature of about $7,500 \mathrm{~K}$, approximately corresponding to CIE standard illuminant $\mathrm{C}$. Its luminance was $57 \mathrm{~cd} / \mathrm{m}^{2}$.

By changing the observer's viewing distance while keeping the stimulus display fixed in place, we varied the angular stripe width of the grating bars in seven steps from $2^{\prime}(15 \mathrm{cpd})$ to $20^{\prime}(1.5 \mathrm{cpd})$. This procedure entailed that the number of bars in the grating stayed constant (in total 24) while the overall width $(7.5 \mathrm{~cm})$ of the grating varied from $1.15^{\circ}$ to $11.5^{\circ}$ and the overall height $(10 \mathrm{~cm})$ varied from $1.53^{\circ}$ to $15.3^{\circ}$.

The gratings were always oriented vertically and were always shown on a gray (Munsell N6) surround. During construction of the gratings, great care was taken to paste and align the Munsell papers exactly. The finished stimuli were then carefully inspected to ensure that no peeled-off parts, shadows, or finger marks were conspicuously present. Some tiny imperfections could not be avoided, but these were deemed too small to affect the subjects' judgments.

\footnotetext{
Subjects

There were 5 observers, all of whom were emmetropes with normal color vision as indicated by the Farnsworth-Munsell 100-Hue Test.

\section{Procedure}

The task of the observer was to choose from a set of 20 Munsell color swatches (plus gray) the color that most clearly matched the perceived hues of the grating bars. The observer alternately viewed the grating and the samples. There was no fixation point. The Mun-
}

sell color swatches were $7.5 \mathrm{~cm}$ in width $\times 10 \mathrm{~cm}$ in height (i.e., they had the same dimensions as the grating stimuli). They were mounted directly above the grating stimuli and were arranged according to the color circle in steps of 5 Munsell hue units. The entire set of matching samples was always on display. The viewing distances (and sizes) of the matching samples and the grating stimuli were therefore concomitantly varied throughout the experiments.

All of the swatches had a Munsell value/chroma notation of $6 / 6$. Thus the effect only of hue, and not of saturation and lightness, was measured. Before beginning the experiment, the observers were first trained to match the colors of the grating with the Munsell swatches and to use visual interpolations when necessary.

During the experiment, the grating color combination and the observation distance were changed in a random order. While such changes were being made, the stimulus display was covered to avoid carryover effects due to persistence or preexposure. Each grating at a given observation distance was presented two separate times. Each subject participated in two $2-h$ sessions.

\section{RESULTS}

Figures 1-3 show the average results of the color matches for the six gratings. Each data point is the average of two measurements for each of 5 subjects. The horizontal bars represent the standard error of the means [i.e., the standard deviation/(the number of observations - 1)]. Hue shifts were found for all color combinations. However, the amount of the shift for a given color depended on the color with which it was paired. For instance, in the yellow/blue grating (Figure 1, top) blue appeared greenish; it had therefore changed in appearance in the direction of the other grating color, yellow. This is an example of hue assimilation. For the finest grating bars ( 2 ' of visual angle), the shift amounted to more than 5 Munsell hue steps, corresponding to a shift in dominant wavelength of about $60 \mathrm{~nm}$. Unlike the blue bars in the grating, the yellow bars displayed only slight variations in hue as a function of stripe width; for stripe widths below 6', they even showed a mild hue contrast.

In the green/blue grating (Figure 1, bottom), the blue bars showed little hue shift, except for the narrowest stripes, which shifted in appearance toward blue-green (i.e., they showed hue assimilation), and the widest stripes, which showed hue contrast. The green bars also appeared blue-green and therefore also displayed hue assimilation. However, they did so much more saliently. Again, the hue shift depended on bar width: for a bar width of $7.5^{\prime}$, no hue assimilation was found. On the other hand, for a bar width of $10^{\prime}$, slight contrast was obtained.

When combined with red bars, green bars displayed assimilation (Figure 2, top): their hue shifted toward yellowgreen. With increasing stripe width, this assimilation was weakened, and at a bar width of $4.5^{\prime}$ it disappeared. Wider bars showed a slight change in the direction of hue contrast. Likewise, the red bars showed assimilation by shifting toward yellow-red. At 6', however, the assimilation disappeared. In the yellow/green grating (Figure 2, bottom), green again showed assimilation. The effect was largest for bar widths of $3^{\prime}$ and $4.5^{\prime}$, and was still present 

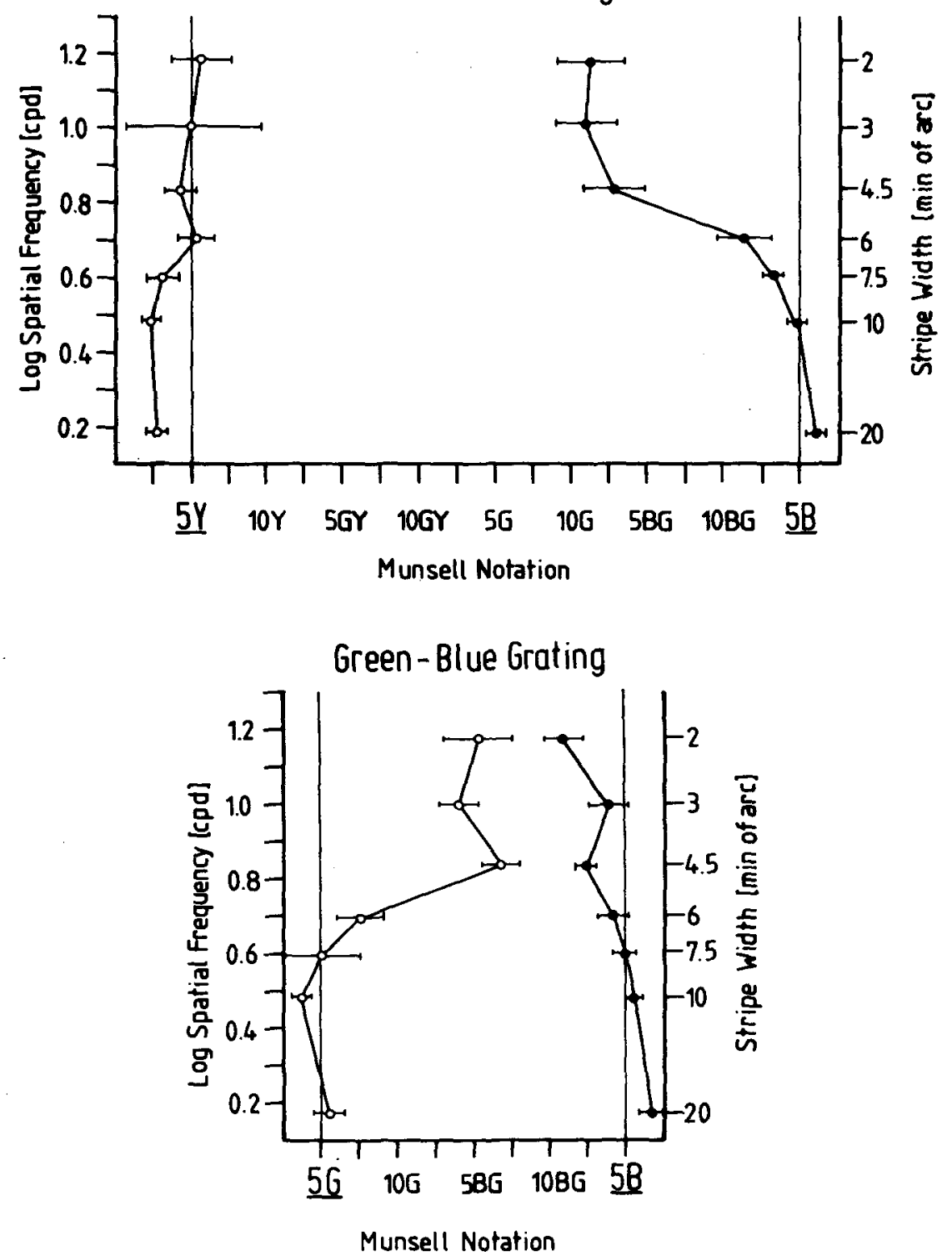

Figure 1. Average results of color matches for a yellow/blue grating (top) and a green/blue grating (bottom). On the left ordinate, $\log$ spatial frequency is plotted (in cpd); on the right ordinate, the corresponding stripe width (in min of arc or visual angle) is given. Munsell notations are plotted on the abscissa. The thin vertical lines indicate the actual Munsell notations of the grating colors. The horizontal bars represent the standard errors of the mean matches.

at 7.5' . At 10', it disappeared. Yellow assimilated for the finest stripes, and displayed contrast for stripes wider than 7.5'.

When blue and red were paired (Figure 3, top), both shifted toward each other (i.e., they assimilated); the effect was largest for the narrowest bars, and diminished as the bar width increased. Likewise, when red and yellow were paired, they both displayed assimilation for the fine stripes (Figure 3, bottom). However, for red, the amount of the shift varied as a function of stripe width, and the widest red stripes even showed contrast, whereas for yellow the amount of shift stayed constant and was always small in magnitude.

Thus, all colors displayed strong assimilation except yellow, which showed little effect in all combinations. In the yellow/blue grating (Figure 1, top), yellow showed no assimilation for bars up to $6^{\prime}$, and for wider bars it displayed a small contrast effect. When combined with green (Figure 2, bottom), yellow showed assimilation only for the narrowest stripes. In combination with red 
(Figure 3, bottom), yellow showed a somewhat stronger assimilation, but unlike that of the other colors, this shift was independent of stripe width.

It should be noted that in none of the color combinations could strong contrast be obtained over the range of stripe widths used. This is made clear in Figure 4, which qualitatively summarizes the data from Figures 1-3. In this figure, the thin and thick arrows represent hue shifts for coarse and fine gratings, respectively. The very slight contrast effects are too small in magnitude to be shown.

\section{DISCUSSION}

On the basis of these results the questions posed at the beginning can now be answered. Strong assimilative color interactions occur between gratings of equal luminance and saturation, but correspondingly strong color contrast interactions do not. The size of the assimilative effects varies considerably, from 1 to 6 Munsell hue steps, with greater hue shifts occurring for the finer stripe widths. However, the amount of the shift depends on the grating color combination used. It is greatest for blue in combination with yellow and for green in combination with blue, and smallest for yellow in all combinations.

Three points about these findings need to be discussed: the small hue changes for yellow, the lack of color contrast in coarser gratings, and the strong hue assimilation in narrow-striped gratings.

For the observation that yellow behaves very differently from other colors when paired in gratings, we can offer
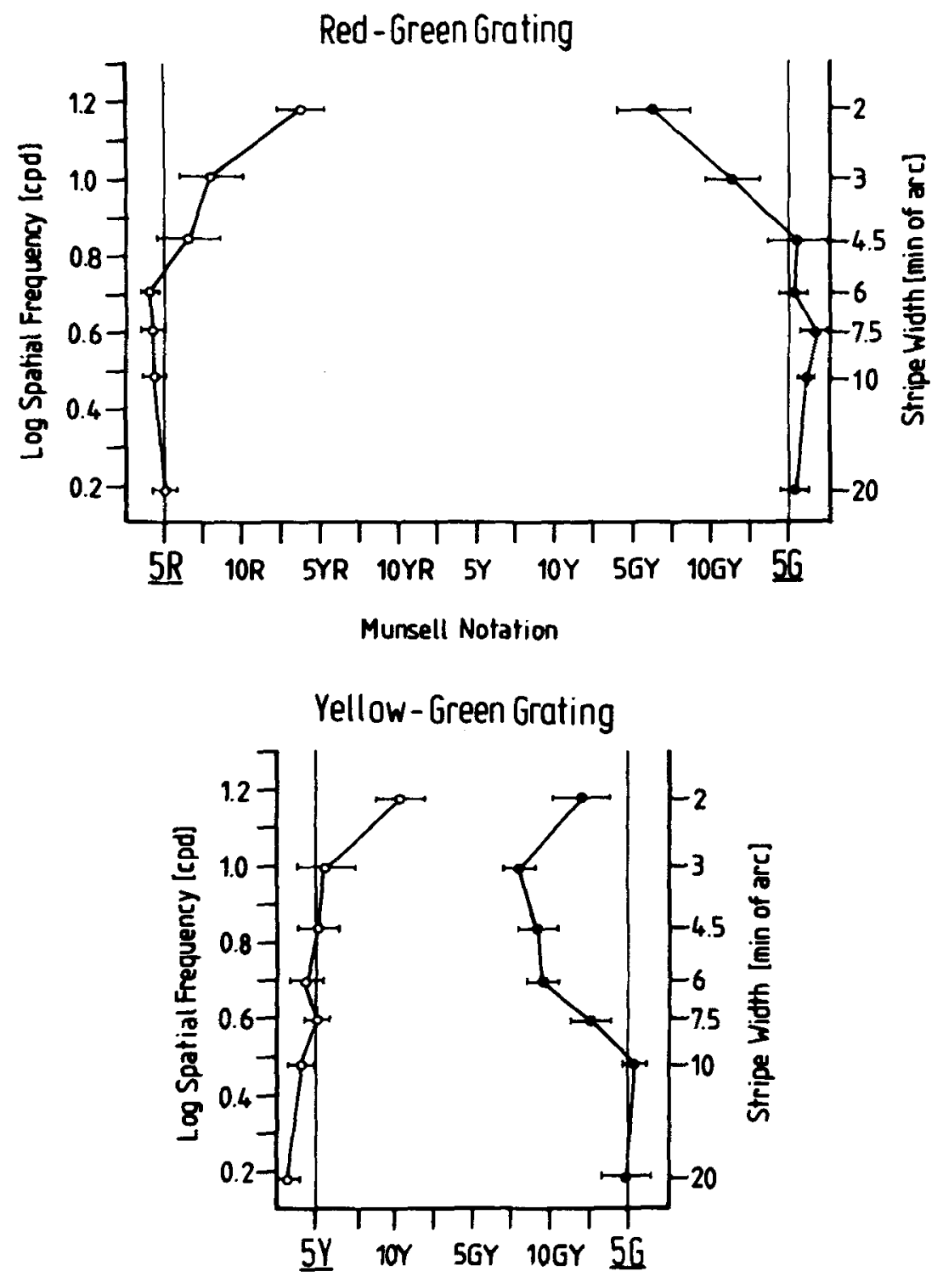

Munsell Notation

Figure 2. Same as Figure 1, for a red/green (top) and a yellow/green (bottom) grating. 


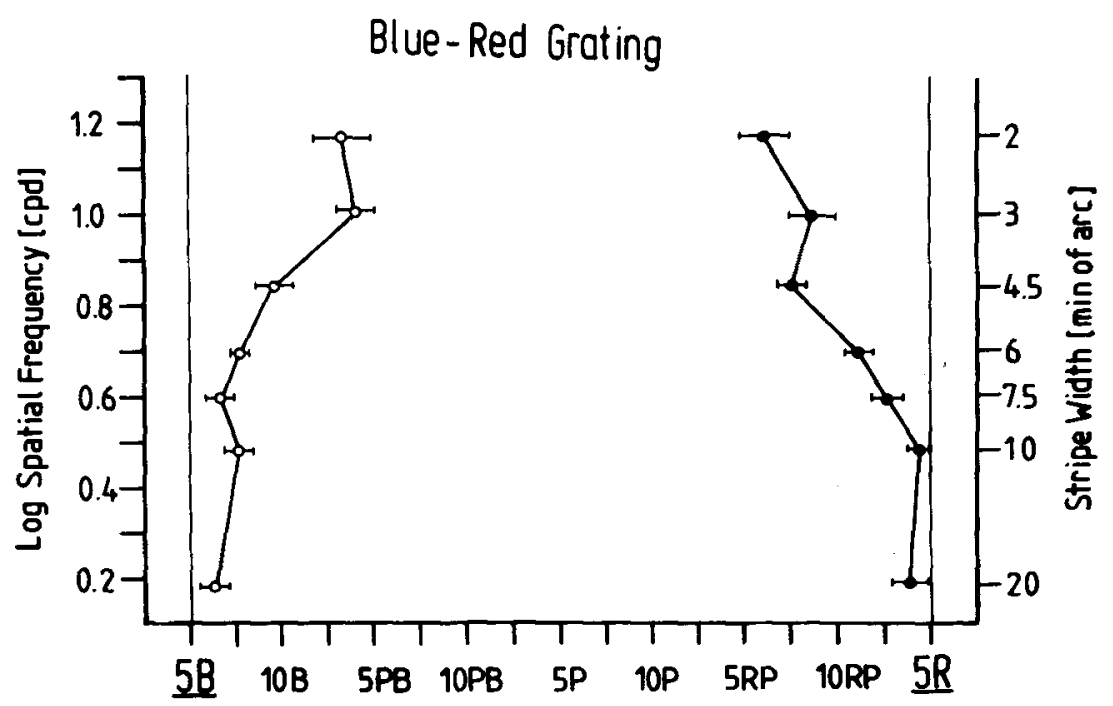

Munsell Notation

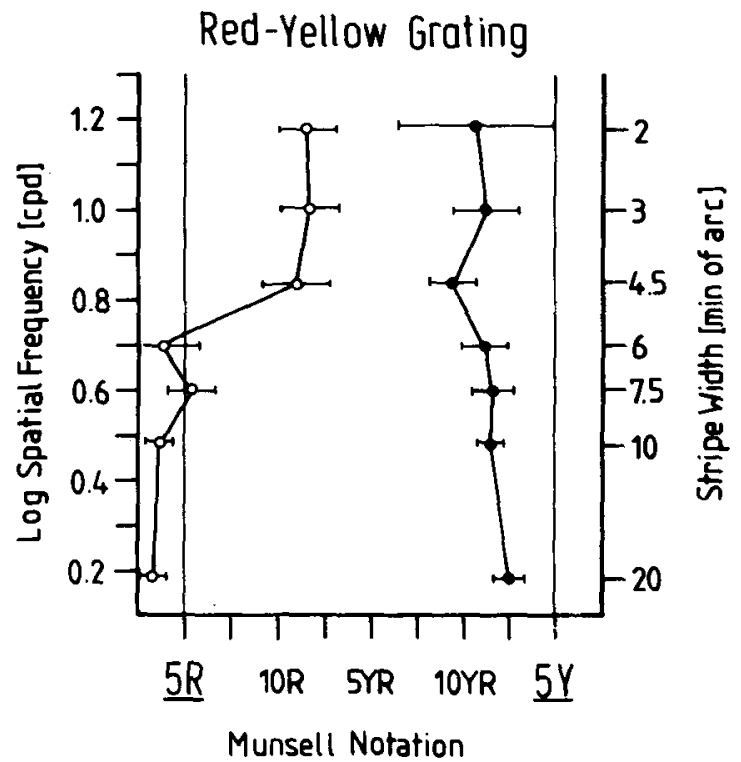

Figure 3. Same as Figure 1, for a blue/red (top) and a red/yellow (bottom) grating.

no satisfactory explanation. We can only point out, first, that the yellow Munsell paper we chose, 5Y (chroma 6), did not appear strongly yellow at any of the value levels used (N1-N6) (in fact, its appearance ranged from sand to ochre); and second, that the peculiar behavior of yellow is not an isolated phenomenon. It is also observed, for example, in the hue constancy of yellow in the Abney effect (see Kurtenbach, Sternheim, \& Spillmann, 1984).

The relative absence of color contrast in the findings presented here is puzzling in view of the fact that such contrast effects have been reported before for chromatic gratings (Ware \& Cowen, 1982). Two factors, however, may be responsible for the failure to find strong color contrast in the present study: the spatial configuration and the saturation of the stimuli. Ware and Cowen may have found color contrast because they used optimal conditions for inducing it. They used inducing stripes of the greatest possible saturation and of greater visual angle than the test stripes ( $18^{\prime}$ vs. $\left.6^{\prime}\right)$. Strong contrast induction would therefore be expected (Helson \& Joy, 1962; Valberg, 1974). In the present study, on the other hand, the inducing and test stripes were comparatively lower in saturation and had the same width. Consequently, less contrast would be expected. Another possible reason for our finding only slight color contrast is that some of our color 


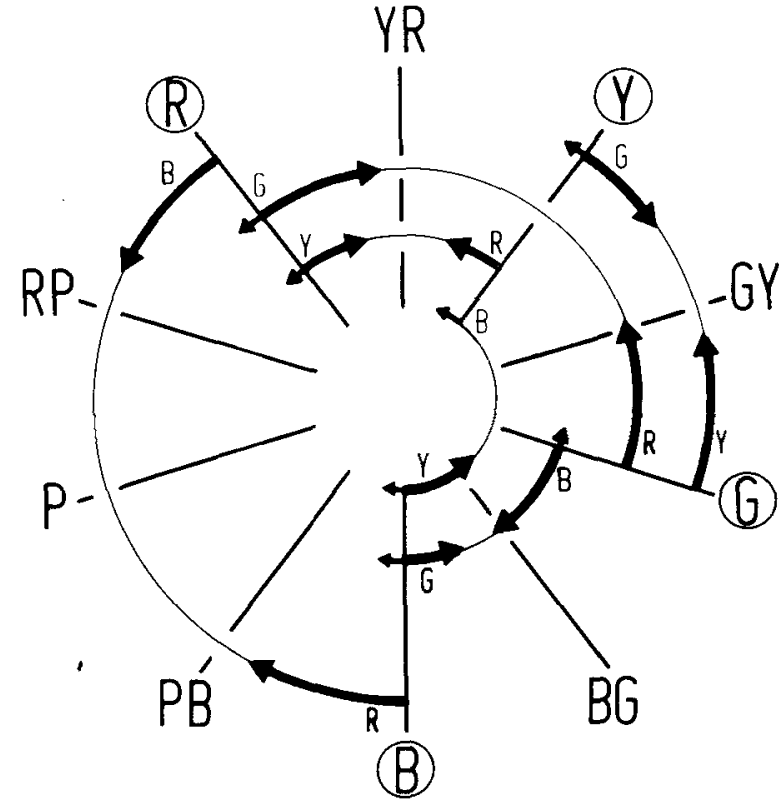

Figure 4. Schematic diagram summarizing the results of Figures 1-3. Thick and thin arrows represent hue shifts for fine and coarse gratings, respectively. The hue notation opposite the arrowhead indicates the inducing color. The length of each arrow approximately represents the magnitude of the assimilative hue shift. Purple was not used in our experiments, but is included in the color diagram for completeness.

pairs, particularly 5Y and 5B, were nearly complementary to each other. Thus, any contrast effects were probably manifested as shifts in saturation, not as shifts in hue. Inasmuch as we measured only hue changes, we failed to detect such contrast effects. In the future, therefore, experiments should be undertaken to investigate systematically the roles of duty cycle and stripe width in perceived hue shifts. Moreover, experiments are required in which perceived shifts not only in hue, but also in lightness and saturation, are simultaneously measured, as was done by Ware and Cowen (1982).

To explain why strong hue assimilation is found for narrow-striped gratings, we must first consider the effects of various physical and physiological factors. These include chromatic aberration, luminance edges, involuntary eye movements, and field size.

The first of these, the axial chromatic aberration of the eye, can be quickly eliminated as a significant factor. It is unlikely to contribute importantly because the Munsell papers have complex, continuous, spectral reflectance curves. Almost all visible wavelengths are present in each paper, and the dominant wavelength does not necessarily correspond to the peak of the reflectance curve. Moreover, the strongest assimilation effects occur in a direction opposite that predicted by chromatic aberration. For instance, short wavelengths are more strongly affected by chromatic aberration than are middle or long wavelengths. Thus, in the yellow/blue grating (Figure 1), light from the blue bars would be expected to overlap and mix with light from the yellow bars, thereby changing the latter's appearance toward blue or, in the case of cancellation, toward gray. But in fact, the blue bars display more assimilation than their yellow counterparts. Furthermore, strong assimilation is found for those color combinations in which the aberration is quite small, for example, red/yellow (Figure 3).

The second factor, the lack of luminance edges in the gratings, can also be largely discounted. Without these edges, accommodation is poor (Wolfe, 1983), and, consequently, the retinal image may be smeared. However, the color mixing or smearing resulting from the use of equiluminant grating bars cannot explain the present findings, because hue shifts should be roughly symmetrical, and this was not the case in the present study.

The third factor, involuntary eye movements, similarly fails to explain our findings. One could argue that such eye movements transfer afterimages onto adjacent stripes and thereby influence the perceived color of those stripes. Again, however, the resulting hue shifts should be symmetrical for both grating colors. As evidenced above, this was not found to be the case.

The fourth factor, the field size, cannot be so easily discounted. This is because we varied spatial frequency by changing the observer's viewing distance. This procedure had the advantage of keeping the total number of bars constant. Thus it controlled for the effect of stimulus redundancy on the potency of assimilation and contrast. However, the procedure allowed the field area to vary over a range of 100:1. Therefore, it might reasonably be argued that some of the observed phenomena resulted not only from the effect of varying spatial frequency, but also from the effect of concomitantly varying field size. This is plausible for three reasons.

First, it is well known that field size has an effect on the precision of color matching: Observers can repeat color matches more precisely when they are obtained in large fields $\left(10^{\circ}\right)$ than when they are obtained in small fields $\left(2^{\circ}\right)$, under otherwise identical observing conditions (Wyszecki \& Stiles, 1982). However, such differences have been found for small, concentric, color-matching stimuli and not for more complex grating stimuli in which the field size is much larger than the stripe width. Thus it is unlikely that the difference between the largest $\left(11.5^{\circ} \times 15.3^{\circ}\right)$ and the smallest $\left(1.15^{\circ} \times 1.53^{\circ}\right)$ field sizes, per se, produced our phenomena.

Second, field size could have confounded the results due to the inhomogeneous distribution of the macular pigment across the retina. This pigment is most dense in the foveal region and trails off in the periphery. Thus, for the highest spatial frequencies (viz., the smallest field sizes), the grating was almost entirely screened by the macular pigment, whereas for the low spatial frequencies (viz., the largest field sizes), only the central portion of the grating was screened by the macular pigment. Since the pigment absorbs maximally at a wavelength of about $460 \mathrm{~nm}$ (Wyszecki \& Stiles, 1982), it acts like a filter to reduce the effect of short-wave stray light. Thus, for our low-spatial-frequency gratings more short-wavelength light reached the peripheral photoreceptors. This could 
considerably alter the phenomena, especially those involving the 5B Munsell grating combinations. Indeed, it is true that the lower frequency gratings with the 5B Munsell paper (see Figure 1, top and bottom, and Figure 3, top) shift in the direction of greater blueness. However, we think it unlikely that the differing extents to which the peripheral retina was stimulated could have significantly contributed to the phenomena, not only because the subjects were instructed to always base their responses on the central portion of the gratings, but also because they were not required to use strict foveal fixation. Discrimination in the blue-green region of the spectrum becomes poor only when very small fields $\left(1^{\prime}-2^{\prime}\right.$ in total extent) are used and when strict foveal fixation is maintained (Bedford \& Wyszecki, 1958; Wyszecki \& Stiles, 1982).

Third, field size could have affected the results due to the changes in distribution of the blue or short-wavesensitive cones with retinal eccentricity. The blue cones have a minimum in the central fovea and a maximum about $1^{\circ}$ to $2^{\circ}$ in the parafovea (Williams, MacLeod, \& Hayhoe, 1981a, 1981b). Moreover, their distribution varies systematically throughout the entire retina, with discrete sensitive peaks being separated by large insensitive gaps (Williams et al., 1981a). However, it is unlikely that such spatial inhomogeneities could have significantly affected our results, because most of our grating stimuli had bars well above the acuity limit of the blue cones, which is about 12-16 cpd for luminance gratings (Stromeyer, Kranda, \& Sternheim, 1978). At any rate, the fundamental limit on resolution is imposed not by the spacing of the receptors, but by other physical factors (Williams, Collier, \& Thompson, 1983). Only for the very smallest grating spatial frequencies used (15 cpd) is it conceivable that the resolution limit of the blue cones could have significantly contributed to assimilation.

For the reasons given above, we feel that the assimilation phenomena reported here are not explained merely by chromatic aberration, eye movements, macular pigment, or field size. Rather, we feel that they are better explained in terms of the receptive field organization of the visual system. This idea also occurred to Hurvich and Jameson (1974), who proposed a model based on the physiological observations that receptive field sizes vary from the periphery to the fovea and show considerable variation in any given retinal region.

Hurvich and Jameson (1974) argued that when a grating pattern is imaged on the retina, neurons that have large receptive fields will integrate over a number of stripes and will respond as if the light were actually mixed on the retina, whereas neurons that have small receptive fields, at the same patch of the retina, will resolve the individual stripes and maintain the spatial resolution of the linear pattern. Thus, the net result for fine grating stripes, but not for coarse ones, will be a blending of hues and brightnesses (assimilation), but a preserving of spatial pattern resolution.

This general model of assimilation, which does not include contrast, may be made more specific by the attri- bution of double-opponent properties to the responsible neurons (see Michael, 1978a, 1978b, 1978c). Such an expanded version of the model can be used to explain why hue shifts are obtained for nonopponent colors, such as green and blue (see Figure 1), and why hues remain constant when the bar width exceeds the receptive field center's size (see Hurvich, 1977, 1981, for more details).

\section{REFERENCES}

BEDFORD, R E., \& WYSZECKI, G. (1958). Wavelength discrimination for point sources. Joumal of the Optical Society of America, 47, 564

CheVreul, M. E. (1969). De la loi de contraste simultane des couleurs. Paris: Leon Laget. (Original work published 1835)

DeVAloIs, R., DeVALoIs, K. (1975). Neural coding of color. In E. C. Carterette \& M. P. Friedman (Eds.), Handbook of perception (Vol. 5, pp. 117-166). New York: Academic Press.

HeLson, H., JoY, V. L. (1962). Domains of lightness assimilation and contrast. Psychologische Beiträge, 6, 405-415.

Hurvich, L. M. (1977). Two decades of opponent processes. In G. Wyszecki \& B. F. Billmeyer, Jr. (Eds.), Color 77, International Color Association (pp. 33-61) New York: Adam Hilger.

Hurvich, L. M. (1981). Color vision. Sunderland, MA: Sinauer Associates.

HURVICH, L. M., JAMESON, D. (1974). Opponent processes as a model of neural organization. American Psychologist, 29, 88-102.

Kurtenbach, W., Sternheim, C. E., \& Splllmann, L. (1984). Change in hue of spectral colors by dilution with white light (Abney effect). Journal of the Optical Society of America, A,1, 365-372.

MichaEL, C. R. (1978a). Color vision mechanisms in monkey striate cortex: Dual opponent cells with concentric receptjve fields. Journal of Neurophysiology, 41, 572-588.

MiCHAEL, C. R. (1978b). Color vision mechanisms in monkey striate cortex: Simple cells with dual opponent-color receptive fieids. Journal of Neurophysiology, 41, 1233-1249.

MiCHAEL, C. R. (1978c). Color sensitive complex cells in monkey striate cortex. Journal of Neurophysiology, 41, 1250-1266.

SCHOBER, H., \& MuNKER, H. (1967). Untersuchungen zu den Übertragungseigenschaften des Gesichtsinns für die Farbinformation. Vision Research, 7, 1015-1026.

Stromeyer, C. F., Kranda, K., Sternheim, C. E. (1978). Selective chromatic adaptation at different spatial frequencies. Vision Research, 18, 427-438.

VAlberg, A. (1974). Color induction: Dependence on luminance, purity, and dominant or complementary wavelength of inducing stimuli. Journal of the Optical Society of America, 64, 1531-1540.

von Bezold, W. (1874). Die Farbenlehre. Braunschweig, West Germany: Westermann.

WALKER, J. T. (1978). Brightness enhancement and the Talbot level in stationary gratings. Perception \& Psychophysics, 23, 356-359.

WARE, C., \& COWEN, W. B. (1982). Changes in perceived color due to chromatic interaction. Vision Research, 22, 1353-1362.

Williams, D. R., Collier, R. J., Thompson, B. J. (1983). Spatial resolution of the short-wavelength mechanism. In J. D. Mollon \& L. T. Sharpe (Eds.), Colour vision, physiology and psychophysics (pp. 487-503). London: Academic Press.

Williams, D. R., MacLeod, D. I. A., Hayhoe, M. (1981a). Foveal tritanopia. Vision Research, 21, 1341-1356.

Williams, D. R., Macleod, D. I. A., \& Hayhoe, M. (1981b). Punctate sensitivity of the blue-sensitive mechanism. Vision Research, 21, $1357-1375$

WOLFE, J. (1983). Hidden visual processes. Scientific American, 248 , 2, 72-85.

WyszecKI, G., STILES, W.S. (1982). Color science (2nd ed.). New York: Wiley.

(Manuscript received May 28, 1985; revision accepted for publication September 17, 1986.) 